\title{
PCE I: The effects of three reward magnitude shifts
}

\author{
GARVIN McCAIN and JOHN COONEY \\ University of Texas at Arlington, Arlington, Texas 76019
}

\begin{abstract}
Six groups of rats were run in a straight alley. Groups were given 46 trials to large (L) or small (S) reward. This was followed by multiple shifts so that groups given reinforcement patterns LLLL, LSSL, SLSL, SSSS, SLLS, and LSLS were formed. A significant PCE was achieved on the second and third shifts to L. In extinction, groups terminating in L were least resistant to extinction.
\end{abstract}

Since Crespi (1944) first produced what appeared to be a positive contrast effect (PCE) as well as a negative contrast effect (NCE), much research and controversy has been generated. The general technique employed by Crespi and others is to start groups on either large (L) or small (S) reward and shift them to either $\mathrm{S}$ or $\mathrm{L}$ reward. A PCE is achieved when the SL group runs faster than a control LL group. The LS group should run more slowly than an SS control group to achieve a NCE. It is generally accepted that the NCE exists. The results regarding the PCE have been equivocal. Sometimes a significant effect has been found; most of the time there has been either a small effect or no effect at all. Certain techniques, such as delay of reward (Mellgren, 1972; Shanab \& Biller, 1972; Shanab \& Cavallaro, 1975), do produce a reliable PCE.

The reasoning behind the present study (one of a series) started with Capaldi's (1974) reinforcement level principle. Capaldi has only recently introduced this extremely important and powerful concept. The following quotes give a short introduction to his reasoning (a much more fully developed statement has been presented as part of the Arlington Symposium on Learning): "It is suggested that strength of conditioning is always determined by the relationship between the reward that is expected and the reward that is obtained. This relationship determines reinforcement level. More specifically, the view that strength of conditioning is determining by reward magnitude defined physically in terms of weight of incentive, etc. (e.g., Capaldi, 1966), is replaced by the view that strength of conditioning is determined by reinforcement level (obtained reward is, of course, defined physically). Accordingly, if obtained reward is greater than expected reward, then available stimuli will acquire a greater capacity than otherwise to elicit the reaction. However, if obtained reward is smaller than expected reward, then available stimuli will suffer a decrease in their capacity to elicit the reaction (unconditioning). Expected reward is assumed to increase as reward magnitude increases and to increase as reward percentage increases" (Capaldi, 1974).

Following this line of reasoning, it seemed possible that a shift from $L$ to $S$ might represent a shift from $L$ to $S$ - due to the contrast. Similarly, a shift of $S$ to $L$ would be S to Lt. This would seem to predict a NCE and a PCE on the first shift. As indicated earlier, the later result has been elusive. One of the problems could be that the SL shift might not provide sufficient contrast. One possible approach would be to use multiple shifts. Thus, for example, we could have LS- to L+ and provide a greater positive contrast than a single shift.

In Crespi's (1942) paper (Experiment II), a PCE is reported following a double shift (LSL). A close reading of the procedures and his analysis of the data suggest caution in interpreting this result.

Others (Capaldi \& Lynch, 1967; Weinstock, 1971) have used more than one magnitude shift. A PCE was not achieved in either of these studies. The studies differ procedurally from the present studies in at least two important ways. Both studies gave a limited number of preshift acquisition trials (15 and 18) and both studies were run at one trial per day. Gandelman and Trowill (1969) and Wagner and Thomas (1966) have also run double shifts. Both of these were very limited studies in that they ran one or two groups. Their evidence does point toward the possibility of a PCE, but due to their limited scope, the results are far from conclusive.

An approach that combines the reinforcement level principle with an arousal notion is currently being considered. One might assume that there are two factors involved in the production of a PCE or NCE. One could be effective habit strength ( $\mathrm{H}$ - inhibition), the other a positive (elation) or negative (frustration) arousal. It might be further assumed that the effects of elation are much weaker than those of frustration. Thus a NCE would be likelier on a first shift than would a PCE. If, on the other hand, arousal were reduced or held constant, both the NCE and PCE should appear at the same stage.

\section{METHOD}

\section{Subjects}

Thirty-six male Holtzman rats, approximately 60 days old on arrival at the laboratory, were the subjects in this study. All subjects were maintained on ad-lib food and water until they were approximately 90 days old, at which time the study was begun.

\footnotetext{
Apparatus

A plywood straight alley, $160 \mathrm{~cm}$ long and painted mid-gray, was
} 
used. The startbox (SB) was approximately $30.5 \mathrm{~cm}$, and the goalbox (GB) approximately $56 \mathrm{~cm}$ long. Opening the SB guillotine door tripped a microswitch which activated a timer. A photoelectric cell approximately $20 \mathrm{~cm}$ from the GB door stopped the first timer and started a second clock, which was stopped by a second photoelectric cell approximately $30.5 \mathrm{~cm}$ from the first cell (GT).

\section{Procedure}

Subjects were on a fixed $15-\mathrm{g}$ total daily ration; water was ad lib throughout the study. Food received in the alley was deducted from the amount received in the home cage. Deprivation and daily handling were begun 9 days before the first acquisition trials were given. The last 4 days before acquisition, the subjects explored the unbaited alley individually for approximately $5 \mathrm{~min}$ per day. During exploration, all equipment was turned on and guillotine doors were raised and lowered.

Throughout the study, the current and past reward conditions of a particular group will be designated as L (11 45-mg Noyes pellets) or S (1 45-mg Noyes pellet). Thus, Group SLSL would have received acquisition training on $S$ reward, Shift 1 would be $L$. Shift $2 S$, and Shift 3 back to L.

Prior to acquisition training, the subjects were randomly divided into two groups, L and S. Two trials were given the first day of acquisition, four the second day, and five trials per day for the remainder of the study. The acquisition phase consisted of $\mathbf{4 6}$ trials.

Subjects were brought into the running room adjoining the animal storage room in carrying cages with six individual compartments and were given a single trial, in rotation, so that on the five-trial days ITI was approximately $5 \mathrm{~min}$. On each individual trial, the subjects were placed in the SB, and approximately $3 \mathrm{sec}$ later the guillotine door was raised. The guillotine door to the GB was lowered when the subject was inside. Subjects remained in the GB until the reward was consumed, then were immediately removed. During extinction, all subjects remained in the GB for $15 \mathrm{sec}$. Subjects failing to reach the GB within $45 \mathrm{sec}$ were guided and given arbitrary scores of $30 \mathrm{sec}$ RT and $15 \mathrm{sec}$ GT.

Shift 1. At the end of acquisition, the subjects were divided within reinforcement groups to give approximately equal mean running speeds over the last 3 days of acquisition. On this basis. four groups were formed: $\operatorname{LL}(n=6), \operatorname{LS}(n=12$; one subject died later and all his data were discarded), SS $(n=6)$, and SL $(n=12)$. All subjects were given $\mathbf{1 5}$ trials at 5 trials per day during this and subsequent shifts.

Shift 2. Groups LLL and SSS continued on schedule, while Group LS was divided and Groups LSL $(n=6)$ and LSS $(n=5)$ were formed, Likewise SL was split, yielding SLS and SLL ( $n=6$ in each case).
Shift 3. There was no matching or splitting at this point; however, Groups LSL-S, LSS-L. SLL-S, and SLS-L were shifted as indicated. The final resulting groups were LLLL, LSSL, LSLS, SSSS. SLLS, and SLSL ( $\mathrm{n}=6$ in each except LSSL, where $\mathrm{n}=5$ ).

Extinction. Extinction followed the 15 trials of Shift 3 and continued for 15 trials. The usual running procedures were used.

\section{RESULTS}

All analyses and graphs are based on a $\log [(1 / \mathrm{med})$ +1 ] transformation. Only RT will be reported for shifts, since there were no important reliable group differences in any of the GT measures during the shifts.

Each shift was analyzed separately. All $t$ tests used for individual comparisons utilized the mean square within from the analysis of variance as an estimate of the pooled variance. Based on a large literature, we assumed the existence of $\mathrm{NCE}$, and all $t$ tests involving a NCE were one-tailed.

\section{Acquisition}

In order to compare the $\mathrm{L}$ and $\mathrm{S}$ groups prior to the first shift, we analyzed the last 3 days of initial acquisition. The $S$ group ran significantly more slowly than the $\mathrm{L}$ group $(\mathrm{p}<.002)$.

\section{Shift 1}

As may be noted from Figure 1a, Group SL does begin running more slowly than Group LL, but runs somewhat faster over days. There is also a change in the comparisons between Groups LS and SS.

In the overall ANOVA, the group differences did not reach a significant level $(\mathrm{F}=2.74, \mathrm{df}=3 / 31, \mathrm{p}>.05)$ The Days by Groups interaction was significant $(\mathrm{F}=$ $13.00, \mathrm{df}=6 / 62, \mathrm{p}<.001)$. In the individual comparisons, Group SL ran more slowly than Group LL on Day $1(\mathrm{t}=2.03, \mathrm{df}=31, \mathrm{p}<.052)$, did not differ on Day $2(\mathrm{t}=1)$, and was significantly faster than LL on Day $3(\mathrm{t}=2.67, \mathrm{df}=31, \mathrm{p}<.02)$. Group LS ran faster

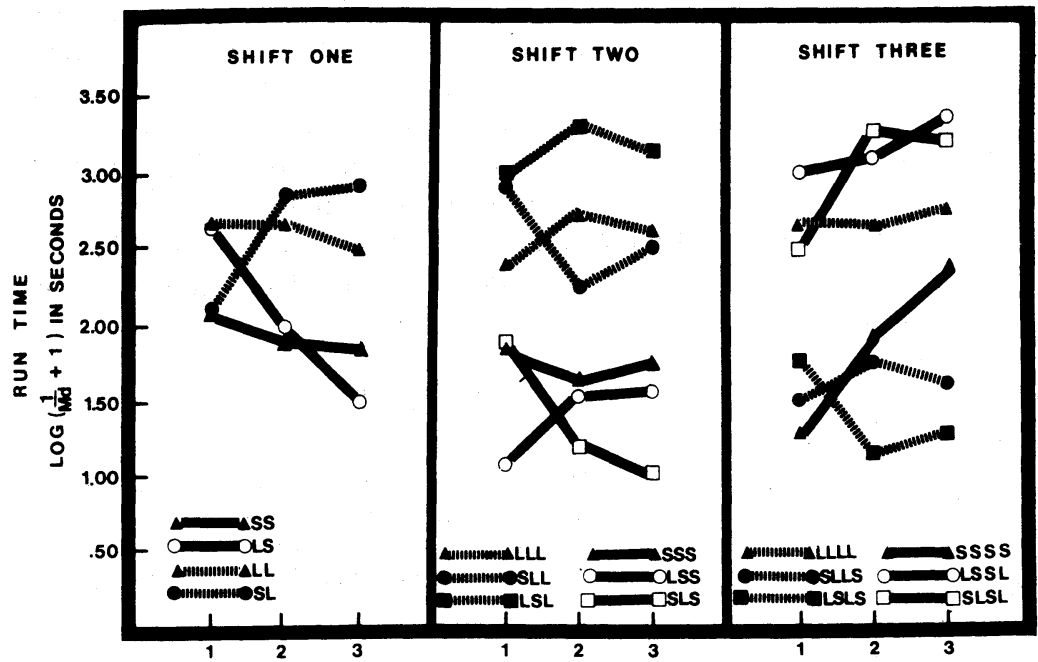

BLOCKS OF FIVE TRIALS
Figure 1 (a, b, c). Terminal acquisition speeds and speeds for each of the three shifts. 
Figure 2. Three days of extinction.

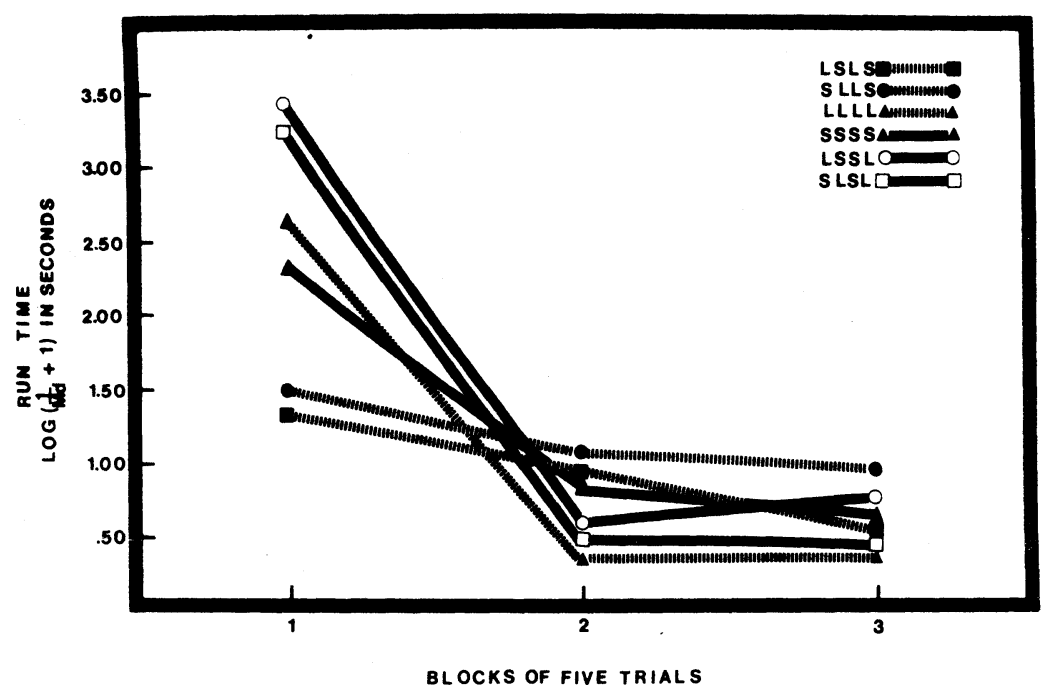

than Group SS on Day $1(\mathrm{t}=3.05, \mathrm{df}=31, \mathrm{p}<.002)$. They did not differ on Day $2(t=.68)$, and LS ran significantly more slowly than SS on Day $3(\mathrm{t}=1.79$, $\mathrm{df}=31, \mathrm{p}<.05)$.

\section{Shift 2}

As may be seen from Figure 1b, Group LSL runs faster than either Group LLL or Group SLL, which appear to be quite similar. Groups SLS and LSS both run more slowly than Group SSS, with Group SLS tending to run more slowly over days in this shift.

In the overall ANOVA, there was a significant groups difference $(F=9.72$, df $=5 / 29, p<.0001)$. In individual group comparisons, Group LSL ran significantly faster than Group LLL $(t=3.19, \mathrm{df}=29, \mathrm{p}<.01)$. Group LSL also ran significantly faster than Group SLL $(t=2.83, \mathrm{df}=29, \mathrm{p}<.01)$. Groups SLL and LLL were very similar $(t=.36)$.

The difference between Groups SSS and SLS was marginally significant. $(\mathrm{t}=1.60 \mathrm{df}=29, \mathrm{p}<.07)$. Groups SSS and LSS were significantly different $(t=$ $1.92, \mathrm{df}=29, \mathrm{p}<.05)$. The difference between Groups SLS and LSS was minimal $(t=.39)$.

\section{Shift 3}

As may be noted from Figure 1c, both Groups LSSL and SLSL appear very similar and each runs faster than Group LLLL. Groups SLLS and LSLS are similar and are somewhat slower than Group SSSS.

In the overall ANOVA, there is a highly significant group difference $(\mathrm{F}=16.82, \mathrm{df}=5 / 29, \mathrm{p}<.0001)$. Group LLLL ran more slowly than either Group SLSL $(\mathrm{t}=2.14, \mathrm{df}=29, \mathrm{p}<.05)$ or Group LSSL $(\mathrm{t}=2.89$, $\mathrm{df}=29, \mathrm{p}<.01)$. Groups $\mathrm{iLSL}$ and LSSL were quite similar $(t=1.28, \mathrm{df}=29, \mathrm{p}<.20>.10)$. Group SSSS ran faster than either Group SLLS $(t=3.30, d f=29$, $p<.002)$ or Group LSLS $(t=3.00, d f=29, p<.005)$. Groups SLLS and LSLS were quite similar $(t=.29)$.

\section{Extinction}

As may be seen in Figure 2, there is a clear distinction between those groups whose terminal reinforcement condition was $L$ and those terminating in $S$ (Terminal Groups S and L). These groups were compared, and there were no significant differences within the individual terminal groups. In the ANOVA, these groups were consolidated based on the terminal reinforcement condition. The groups difference was not significant $(\mathrm{F}=1.75, \mathrm{df}=1 / 30, \mathrm{p}<.10)$. The Group by Days interaction was significant $(F=4.43$, $\mathrm{df}=2 / 66$, $\mathrm{p}<.02)$. Day-by-day comparisons indicated that on Day 1, Terminal Group L ran significantly faster than Terminal Group $S(t=8.65, \mathrm{df}=33, \mathrm{p}<.001)$. However, on both Days 2 and 3, Terminal Group L ran more slowly than Terminal Group $\mathrm{S}(\mathrm{t}=3.35$, $\mathrm{df}=33$, $\mathrm{p}<.001 ; \mathrm{t}=2.19$, df $=33, \mathrm{p}<.05$, respectively). Since Terminal Group $L$ was running faster over the last 15 trials than Terminal Group $S$ at the beginning of extinction $(\mathrm{F}=70.37, \mathrm{df}=1 / 33, \mathrm{p}<.0001)$, extinction was also calculated using each subject as his own base line. There is an overlap of only one animal between the groups, and the difference is highly significant $(\mathrm{F}=65.53, \mathrm{df}=1 / 33, \mathrm{p}<.0001)$, with Terminal Group $\mathrm{L}$ the slower.

\section{DISCUSSION}

Although our original reasoning started with the reinforcement level principle, the extinction phase of the present study does raise some serious questions.

For the moment, the present study should be considered primarily for its empirical value. It does seem to represent a reliable and replicable method of producing a PCE without the possible confounding of delay or other additional variables.

The fact that a significant PCE was obtained on Day 3 of Shift 1 may be related to several factors. It may be that Group SL was increasing in speed and would have eventually shown a PCE. However, Group SLL in Shift 2 represents a continuation of a matched half of Group SL and did not achieve a PCE in Shift 2 , 
which argues against the PCE before Shift 2. Also, Group LL ran more slowly on Day 3 of Shift 1 than on either Day 1 or Day 2, and the significant difference from Group SL on Day 3 may be an artifact.

The data presented from the shifts in this study could be analyzed in terms of the reinforcement level hypothesis (Capaldi, 1974). A succeeding study suggests extreme caution. In the later study, four shifts were used. In Shift 4. training groups shifted to $S$ ran faster than the $S$ control group. If this sort of result is confirmed, the analysis will be quite different than the present data alone suggests.

It may be useful to present the present data alone rather than waiting for analysis and interpretation of the succeeding studies. First. the scope of this study is limited and will probably support. but not add to, the succeeding studies. Second, the multiple shift as a technique may lead others to some interesting findings. For example. delay of reinforcement and schedules of reinforcement suggest themselves as areas where the results of single shifts may not necessarily be the same as results from multiple shifts.

The extinction data are of some interest. The general finding was that after multiple shifts, $R$ to $E$ was a function of the last reinforcement schedule. Groups LSSL, SLSL. and LLLL (Terminal Group L) were quite similar in performance, as were Groups SSSS, SLLS, and LSLS (Terminal Group S); further, these terminal groups differed from one another. with Terminal Group $L$ being less $R$ to $E$. The number of $S$ or $L$ trials appears unrelated to $R$ to $E$ over the last 2 days of extinction. For example, both SLSL and SLLS have $61 \mathrm{~S}$ and $30 \mathrm{~L}$ trials, yet SLLS is the most $\mathrm{R}$ to $\mathrm{E}$ while SLSL is next to least $R$ to $E$ of the six groups. S-to-L and L-to-S transitions were also considered, but no systematic relation could be found. These results do not seem to be predicted from the reinforcement level principle (Capaldi. Note 1). The results to date are so limited that no clear conclusions are justified. Birch and Valle's (1967) finding that $R$ to $E$ decreased as the number of $L$ trials increased for groups given a single shift is not necessarily incompatible with the present finding.

As indicated earlier. delay of reinforcement has been used during acquisition to produce a PCE when subjects are shifted from small to large reward. One reason for the use of delay is to slow down the subjects and thus avoid a ceiling effect. The present study indicates that at least under these conditions the ceiling presents no great problem.

\section{REFERENCE NOTE}

1. Capaldi. E. J. Reinforcement level: An expectancy-associative approach to relutive reinforcement and nonreinforcement effects. Arlington Symposium on Learning. University of Texas at Arlington. February 1975.

\section{REFERENCES}

Birch. D..\& VALLE, F. P. Resistance to extinction in the runway following a shift from small to large reward. Journal of Comparative and Physiological Psychology, 1967, 63. 50-53.

CAPAldr. E. J. Partial reward either following or preceding consistent reward: A case of reinforcement level. Journal of Experimentul Psychology. 1974. 102. 954-962.

CAPAldi. E. J.. \& LYNCH. D. Repeated shifts in reward magnitude: Evidence in favor of an associational and absolute (noncontextual) interpretation. Journal of Experimental Psychology. 1967. 75. 226-235.

CREspi. L. P. Quantitive variation of incentive and performance in the white rat. American Journal of Psychology. 1942. 75. 467.517 .

Crespi. L. P. Amount of reinforcement and level of performance. Psichological Review, 1944. 51. 341-357.

Gandelman. R.. \& Trowill. J. A. Effects of reinforcement shifts upon subsequent saccharin consumption. Psychonomic Science. 1969. 15. 25.

Mellgren. R. L. Positive and negative contrast effects using delaved reinforcement. Learning and Motivation. 1972, 3. $1856-193$.

Shanab. M. E.. \& Biller, J. D. Positive contrast in the runway obtained following a shift in both delay and magnitude of reward. Learning and Motivation. 1972, 3. 179-184.

Shanab. M. E.. \& Cavallaro. G. Positive contrast obtained in rats following a shift in schedule. delay and magnitude of reward. Bulletin of the Psychonomic Society, 1975. 5. 109-112.

WAGNer. A. R.. \& Thomas. E. Reward magnitude shifts: A savings effect. Psychonomic Science. 1966, 4. 13-14.

WEINSTOCK. R. B. Preacquisition exploration of the runway in the determination of contrast effects in the rat. Journal of Comparative and Physiological Psychology. 1971. 75. 107-115. 